\title{
EXTENDIBILITY CRITERION FOR A PROJECTIVE MODULE OF RANK ONE OVER $R[T]$ AND $R\left[T, T^{-1}\right]$
}

\author{
S. M. BHATWADEKAR AND P. L. N. VARMA
}

(Communicated by Louis J. Ratliff, Jr.)

\begin{abstract}
In this note we give a criterion for a finitely generated projective module $\mathscr{P}$ of constant rank one over $R[T]$ or $R\left[T, T^{-1}\right]$ to be extended from $R$ in terms of invertible ideals, when $R$ is an integral domain. We show that if $I$ is an invertible ideal of $R[T]$ or $R\left[T, T^{-1}\right]$ such that $I \cap R \neq 0$, then $I$ is extended from $R$ if and only if $I \cap R$ is an invertible ideal of $R$.
\end{abstract}

\section{INTRODUCTION}

Let $R$ be a commutative ring, and let $A$ be the polynomial algebra $R[T]$ or the Laurent polynomial algebra $R\left[T, T^{-1}\right]$. Let $\mathscr{P}$ be a finitely generated projective $A$-module. We say that " $\mathscr{P}$ is extended from $R$ " if there exists an $R$-module $\mathscr{Q}$ such that $\mathscr{P} \simeq \mathscr{Q} \otimes_{R} A$ as $A$-modules. In this note we investigate the question: when is a finitely generated projective module $\mathscr{P}$ of (constant) rank one over $A$ extended from $R$ ? It is easy to see that for this question we can assume without loss of generality that $R$ is a reduced ring. Hence, throughout the paper we will assume that $R$ is a reduced commutative ring.

If $R$ has only finitely many minimal prime ideals (e.g., $R$ is an integral domain or $R$ is a noetherian ring) then $Q(R)$, the total quotient ring of $R$, is a finite direct product of fields. In this case, since all finitely generated projective modules of (constant) rank one over $Q(R)[T]$ and $Q(R)\left[T, T^{-1}\right]$ are free, it is easy to see that there exists an invertible ideal $I$ of $A$ such that

(1) $I \cap R$ contains a non-zero-divisor of $R$,

(2) $I \simeq \mathscr{P}$ as $A$-modules.

See [1, Chapter II, $\S 5]$ for details. Therefore, in this situation, one is reduced to consider the following question:

Question. Let $R$ be a reduced ring with only finitely many minimal prime ideals. Let $A$ denote the polynomial algebra $R[T]$ or the Laurent polynomial algebra $R\left[T, T^{-1}\right]$. Let $I$ be an invertible ideal of $A$ such that $I \cap R$ contains a non-zero-divisor of $R$. Then, when is $I$ extended from $R$ ?

In this paper we settle this question as follows:

Received by the editors September 26, 1991 and, in revised form, April 6, 1992.

1991 Mathematics Subject Classification. Primary 13C10; Secondary 13F20. 
Theorem (A). Let $R$ be a reduced ring with only finitely many minimal prime ideals. Let $I$ be an invertible ideal of $R\left[T, T^{-1}\right]$ such that $J=I \cap R$ contains a non-zero-divisor of $R$. Then $I$ is extended from $R$ if $J$ is an invertible ideal of $R$. Moreover, if $R$ is an integral domain and $I$ is extended from $R$, then $J=I \cap R$ is an invertible ideal of $R$.

Theorem (B). Let $R$ be a reduced ring with only finitely many minimal prime ideals. Let $I$ be an invertible ideal of $R[T]$ such that $J=I \cap R$ contains a nonzero-divisor of $R$. Then $I$ is extended from $R$ if and only if $J$ is an invertible ideal of $R$.

We also give an example of a reduced noetherian ring and an invertible ideal $I$ of $A=R\left[T, T^{-1}\right]$ such that $J=I \cap R$ contains a non-zero-divisor of $R, I$ is extended from $R$ as an $A$-module, but $J$ is not an invertible ideal of $R$.

In case of an ideal $I$ of $A=R[T]$ or $R\left[T, T^{-1}\right]$, there are naturally two notions of extendibility, namely,

(1) ideal-extendibility, i.e., $I=\mathscr{I} A$ for some ideal $\mathscr{I}$ of $R$,

(2) module-extendibility, i.e., there exists an $R$-module $M$ such that $I \approx$ $M \otimes_{R} A$ as $A$-modules.

Obviously ideal-extendibility implies module-extendibility, but the converse need not be true.

Theorem (A) and Theorem (B) are proved by first showing that if $A=$ $R\left[T, T^{-1}\right]$ ( $R$ a domain) or $A=R[T]$ ( $R$ a reduced ring), then for an ideal $I$ of $A$ the two notions of extendibility are equivalent if $I \cap R$ contains a non-zero-divisor (Lemmas 2.4 and 2.8). Example 2.7 shows that for $A=R\left[T, T^{-1}\right]$, the two notions need not be the same even for an invertible ideal $I$ of $A$ containing a non-zero-divisor of $R$ if $R$ is not a domain.

\section{EXTENDIBILITY CRITERION}

In this section we will prove Theorems (A) and (B) stated above (Theorems 2.11 and 2.13, respectively). We begin with the following definition:

Definition 2.1. Let $A$ be a reduced ring, and let $Q(A)$ denote the total quotient ring of $A$. An $A$-submodule $M$ of $Q(A)$ is said to be invertible if there exists an $A$-submodule $N$ of $Q(A)$ such that $M N=A$.

We note that such an $N$ is unique and we denote it by $M^{-1}$. If an ideal $I$ of $A$ is invertible, we say that $I$ is an invertible ideal of $A$.

Let $B$ be an $A$-subalgebra of $Q(A)$, and let $I$ be an invertible ideal of $A$. Then it follows immediately from the definition that $I B$ is an invertible ideal of $B$.

Now we state a lemma, a proof of which can be found in [1, Chapter II, §5].

Lemma 2.2. Let $A$ be a reduced ring and $S$ be a multiplicative set of non-zerodivisors of $A$. Let $B=S^{-1} A$. If all finitely generated projective $B$-modules of constant rank one are free, then given a finitely generated projective $A$-module $\mathscr{P}$ of constant rank one there exists an invertible ideal $I$ of $A$ such that $I \cap S \neq \varnothing$ and $I \simeq \mathscr{P}$ as A-modules.

As a consequence of the above lemma we have the following: 
Lemma 2.3. Let $R$ be a reduced ring with only finitely many minimal prime ideals. Let $S$ denote the set of all non-zero-divisors of $R$. Let $A=R[T]$ or $R\left[T, T^{-1}\right]$, and let $\mathscr{P}$ be a finitely generated projective $A$-module of constant rank one. Then there exists an invertible ideal $I$ of $A$ such that $I \cap S \neq \varnothing$ and $I \simeq \mathscr{P}$.

Now we prove Theorems (A) and (B) stated in the introduction. For the proof of these theorems we need some lemmas.

Lemma 2.4. Let $R$ be a domain, and let $I$ be a finitely generated ideal of $R\left[T, T^{-1}\right]$. Assume that $J=I \cap R$ is nonzero. Then the following statements are equivalent:

(1) $I=J R\left[T, T^{-1}\right]$.

(2) $I \simeq J \otimes_{R} R\left[T, T^{-1}\right]$ as $R\left[T, T^{-1}\right]$-modules.

(3) There exists an $R$-module $M$ such that $I \simeq M \otimes_{R} R\left[T, T^{-1}\right]$ as $R\left[T, T^{-1}\right]$-modules.

Proof. The implications $1 \Rightarrow 2$ and $2 \Rightarrow 3$ hold for any ring $R$ (not necessarily a domain). So it remains to prove the implication $3 \Rightarrow 1$.

Let $M$ be an $R$-module such that $I \simeq M \otimes_{R} R\left[T, T^{-1}\right]$ as $R\left[T, T^{-1}\right]$ modules. Since $I$ is a finitely generated nonzero ideal of $R\left[T, T^{-1}\right]$ and $R\left[T, T^{-1}\right]$ is a free $R$-module, it follows that $M$ is a finitely generated torsion free $R$-module of rank one. Hence there exists a finitely generated nonzero ideal $\mathscr{I}$ of $R$ such that $M \simeq \mathscr{I}$ as $R$-modules. Thus

$$
\mathscr{J} R\left[T, T^{-1}\right] \simeq \mathscr{J} \otimes_{R} R\left[T, T^{-1}\right] \simeq M \otimes_{R} R\left[T, T^{-1}\right] \simeq I
$$

as $R\left[T, T^{-1}\right]$-modules. Let $\theta: \mathscr{I} R\left[T, T^{-1}\right] \rightarrow I$ be an isomorphism. Let $b \in$ $\mathscr{I}$ be a nonzero element of $R$. Then we claim that $b I=\theta(b) \mathscr{I} R\left[T, T^{-1}\right]$.

Let $g \in I$ and $h \in \mathscr{J} R\left[T, T^{-1}\right]$ be such that $\theta(h)=g$. Then $b g=$ $b \theta(h)=\theta(b h)=h \theta(b)$ and this proves the claim.

Let $c \in I$ be a nonzero element of $R$. Then $c b=\theta(b) f$ for some $f \in$ $\mathscr{I} R\left[T, T^{-1}\right]$. But since $R$ is a domain, this shows that $\theta(b) T^{n}=a \in R$ for some integer $n$. Now the equality $b I=\theta(b) \mathscr{I} R\left[T, T^{-1}\right]=a \mathscr{I} R\left[T, T^{-1}\right]$ gives that $b J=b(I \cap R)=a \mathscr{I}$. Therefore $b I=b J R\left[T, T^{-1}\right]$ and hence $I=J R\left[T, T^{-1}\right]$.

Remark 2.5. Lemma 2.4 is true if $R$ is a finite direct product of domains and $I \cap R$ contains a non-zero-divisor of $R$.

Remark 2.6. The following example shows that Lemma 2.4 need not be true if $R$ is not a direct product of domains.

Example 2.7. Let $R=k \llbracket X, Y \rrbracket /(X Y)=k \llbracket x, y \rrbracket$. Let $f=x+y T$ be an element of $R\left[T, T^{-1}\right]$ and let $I=f R\left[T, T^{-1}\right]$. Then it is easy to see that $I \cap$ $R=\left(x^{2}, y^{2}\right)$, which contains a non-zero-divisor $x^{2}-y^{2}$. Moreover, since $f$ is a non-zero-divisor of $R\left[T, T^{-1}\right]$, the ideal $I$ is a free module of rank one over $R\left[T, T^{-1}\right]$ and hence it is extended from $R$ as an $R\left[T, T^{-1}\right]$-module. But obviously $f R\left[T, T^{-1}\right] \neq\left(x^{2}, y^{2}\right) R\left[T, T^{-1}\right]$ as $\left(x^{2}, y^{2}\right)$ is not an invertible ideal of $R$.

In the case of a polynomial algebra $R[T]$ we get the following generalisation of Lemma 2.4. 
Lemma 2.8. Let $R$ be a reduced ring, and let $I$ be a finitely generated ideal of $R[T]$. Assume that $J=I \cap R$ contains a non-zero-divisor. Then the following statements are equivalent:

(1) $I=J R[T]$.

(2) $I \simeq J \otimes_{R} R[T]$ as $R[T]$-modules.

(3) There exists an $R$-module $M$ such that $I \simeq M \otimes_{R} R[T]$ as $R[T]$ modules.

Proof. As above the implications $1 \Rightarrow 2$ and $2 \Rightarrow 3$ are obvious. Thus it remains to prove $3 \Rightarrow 1$.

Let $s \in J$ be a non-zero-divisor of $R$. Then it is easy to see that $M$ is a finitely generated torsion free $R$-module and $M_{s}$ is a free $R_{s}$-module of rank one. Therefore there exists a finitely generated ideal $\mathscr{I}$ of $R$ such that $\mathscr{I} \simeq M$ as $R$-modules. Moreover, since $\mathscr{I}_{s}$ is a free $R_{s}$-module of rank one, without loss of generality we can assume that $t=s^{n} \in \mathscr{J}$ for some positive integer $n$. Let $\theta: \mathscr{I} R[T] \rightarrow I$ be an isomorphism of $R[T]$-modules. Then we claim that $t I=\theta(t) \mathscr{F} R[T]$.

Let $g \in I$ and $f \in \mathscr{I} R[T]$ be such that $\theta(f)=g$. Then $t g=t \theta(f)=$ $\theta(t f)=f \theta(t)$. This proves the claim.

Since $s \in I$, the equality $t I=\theta(t) \mathscr{J} R[T]$ shows that $t s=\theta(t) g$ for some $g \in \mathscr{I} R[T]$. Now by Lemma 2.9 (stated below) we have $\theta(t) \in R$. Therefore

$$
t J=t I \cap R=\theta(t) \mathscr{I} R[T] \cap R=\theta(t) \mathscr{I} .
$$

Hence $t I=t J R[T]$. But $t$ is a non-zero-divisor of $R$. Therefore $I=$ $J R[T]$.

Lemma 2.9. Let $R$ be a reduced ring and $s$ be a non-zero-divisor of $R$. Let $f \in R[T]$ be such that $s \in f R[T]$. Then $f \in R$.

Proof. Let $s=f(T) g(T)$ for some $g(T) \in R[T]$. Write $f(T)=a_{0}+a_{1} T+$ $\cdots+a_{n} T^{n}$ for some $a_{i} \in R, 0 \leq i \leq n$, with $a_{n} \neq 0$. We want to show that $n=0$.

Since $s=f(0) g(0)=a_{0} g(0)$ and $s$ is a non-zero-divisor, $a_{0}$ is either a unit or a non-zero-divisor in $R$. If $n>0$ then since $R$ is reduced there exists a minimal prime ideal $p$ of $R$ such that $a_{n} \notin p$. Since $a_{0}$ is a unit or a nonzero-divisor, obviously $a_{0} \notin p$. Let 'bar' denote "modulo $p$ ". Then we have $\bar{s}=\bar{f}(T) \bar{g}(T)$ in $\bar{R}[T]$. But since $\bar{f}(T)$ is a polynomial of positive degree this is absurd and hence $n=0$.

When $R$ is reduced (but not necessarily a domain) one has the following weaker version of Lemma 2.4 .

Lemma 2.10. Let $R$ be a reduced ring, and let $I$ be a finitely generated ideal of $R\left[T, T^{-1}\right]$ such that $J=I \cap R$ contains a non-zero-divisor of $R$. If $I$ is extended from $R$ as a module, then there exists an element $f$ of $R\left[T, T^{-1}\right]$ such that $f$ is not a zero-divisor of $R\left[T, T^{-1}\right]$ and $f I$ is extended from $R$ as an ideal.

This easily follows from the proof of Lemma 2.4.

Now we prove the main theorems. 
Theorem 2.11. Let $R$ be a reduced ring with only finitely many minimal prime ideals. Let $I$ be an invertible ideal of $R\left[T, T^{-1}\right]$ such that $J=I \cap R$ contains a non-zero-divisor of $R$. Then $I$ is extended from $R$ if $J$ is an invertible ideal of $R$. Moreover, if $R$ is an integral domain and $I$ is extended from $R$, then $J=I \cap R$ is an invertible ideal of $R$.

Proof. Let $I$ be an invertible ideal of $R\left[T, T^{-1}\right]$ such that $J=I \cap R$ contains a non-zero-divisor $S$ of $R$ and $J$ is an invertible ideal of $R$. If $I=R\left[T, T^{-1}\right]$ then clearly $I=J R\left[T, T^{-1}\right]$, where $J=I \cap R=R$. So we can assume that $I$ is a proper ideal of $R\left[T, T^{-1}\right]$. Now we will show that $I=J R\left[T, T^{-1}\right]$. Clearly $J R\left[T, T^{-1}\right] \subseteq I$ and to show the equality it is enough to show that for every maximal ideal $\mathfrak{I}$ of $R, J_{\mathfrak{I}} R_{\mathfrak{I}}\left[T, T^{-1}\right]=I_{\mathfrak{I}}$. But then $I_{\mathfrak{I}} \cap R_{\mathfrak{I}}=J_{\mathfrak{I}}$, and as $J_{\mathfrak{J}}$ is an invertible ideal in the local ring $R_{\mathfrak{J}}, J_{\mathfrak{J}}$ is principal and hence $J_{\mathfrak{I}}=t R_{\mathfrak{I}}$ for some $t \in R$ which is not a zero-divisor of $R$. Thus by replacing $R$ with $R_{\mathfrak{J}}$ we are reduced to proving the following:

Let $R$ be a reduced local ring with only finitely many minimal prime ideals. Let $I$ be an invertible ideal of $R\left[T, T^{-1}\right]$ which is a proper ideal such that $I \cap R=t R$ for some non-zero-divisor $t$ of $R$. Then $I=t R\left[T, T^{-1}\right]$.

Since $I$ is an invertible ideal of $R\left[T, T^{-1}\right]$ containing a non-zero-divisor $t$ of $R$, it is easy to see that the canonical epimorphism $I /(T-1) I \rightarrow I+$ $(T-1) /(T-1)$ is an isomorphism and hence

$$
I /(T-1) I \simeq I+(T-1) /(T-1) \simeq I / I \cap(T-1) .
$$

This shows that $I+(T-1) /(T-1)$ is an invertible ideal of $R$ which contains the element $t$ of $R$.

If $I \neq t R\left[T, T^{-1}\right]$ then there exists an element $g_{1} \in R\left[T, T^{-1}\right]$ such that $g_{1} \in I \backslash t R\left[T, T^{-1}\right]$. Without loss of generality we may assume that $g_{1}$ is a polynomial in $R[T]$ and is of least degree (among such elements of $I$ ). Let us write $g_{1}$ as $g_{1}=a_{0}+a_{1}(T-1)+\cdots+a_{r}(T-1)^{r}$ with $a_{i} \in R$ and $a_{r} \neq 0$. Obviously $t \nmid a_{0}$. Otherwise $a_{0}=t a$ for some $a \in R$. Hence $g_{1}-a_{0}=g_{1}-t a=(T-1) f$ for some $f \in R[T]$. As $T-1$ is a non-zerodivisor modulo $I$ (by $(*)$ ) we have $f \in I \backslash t R\left[T, T^{-1}\right]$. But $\operatorname{deg} f<\operatorname{deg} g_{1}$, contradicting the minimality of degree of $g_{1}$. Hence $t \nmid a_{0}$.

Let $\left\{t, g_{1}, g_{2}, \ldots, g_{n}\right\} \subseteq R\left[T, T^{-1}\right]$ be a set of generators of $I$, where $g_{i} \in R[T]$ for $1 \leq i \leq n$. Then $I+(T-1) /(T-1)=\left(t, g_{1}(1)=a_{0}, g_{2}(1), \ldots\right.$, $\left.g_{n}(1)\right)$. Since $I+(T-1) /(T-1)$ is an invertible ideal of $R$ and $R$ is local, $I+(T-1) /(T-1)$ is a principal ideal of $R$ generated, say, by $b$ and $b \in$ $\left\{t, a_{0}, g_{2}(1), \ldots, g_{n}(1)\right\}$. But since $t \nmid a_{0}$ we have $t \nmid b$. So $b=g_{i}(1)$ for some $i, 1 \leq i \leq n$. Moreover $t / b=d$ belongs to the maximal ideal of $R$.

Let $\bar{R}$ be the normalisation of $R$ in its total quotient ring. Note that $\bar{R}$ is a finite direct product of domains. Since $\bar{R}$ is normal and $I \bar{R}\left[T, T^{-1}\right]$ is invertible, it is extended from $\bar{R}$ as a module. Therefore, as $I \bar{R}\left[T, T^{-1}\right]$ contains a non-zero-divisor of $\bar{R}$, namely $t$, by Lemma 2.4 and Remark 2.5 , $I \bar{R}\left[T, T^{-1}\right]=L \bar{R}\left[T, T^{-1}\right]$, where $L=I \bar{R}\left[T, T^{-1}\right] \cap \bar{R}$.

Let $\left\{a_{1}, a_{2}, \ldots, a_{m}\right\} \subseteq \bar{R}$ be a set of generators for $L$. Recall that $\left\{t=g_{0}, g_{1}, \ldots, g_{n}\right\}$ is a set of generators for $I \bar{R}\left[T, T^{-1}\right]$. Then we get 
the following relations:

$$
\begin{array}{ll}
g_{i}=\sum_{j=1}^{m} h_{i j} a_{j} & \text { for } i=0,1, \ldots, n, \\
a_{k}=\sum_{l=0}^{n} f_{k l} g_{l} & \text { for } k=1,2, \ldots, m,
\end{array}
$$

for some $h_{i j}$ and $f_{k l}$ in $\bar{R}\left[T, T^{-1}\right]$. Let $R^{\prime}$ be the $R$-subalgebra of $\bar{R}$ generated by $\left\{a_{i}\right\} \cup$ coefficients of $\left\{h_{i j}, f_{k l}\right\}$, and let $L^{\prime}$ be the ideal of $R^{\prime}$ generated by $\left\{a_{1}, a_{2}, \ldots, a_{m}\right\}$. Clearly $R^{\prime}$ is a finitely generated $R$-subalgebra of $\bar{R}$. Therefore $R^{\prime}$ is a finite $R$-module and hence $R^{\prime}$ is semilocal. Then the equality $I R^{\prime}\left[T, T^{-1}\right]=L^{\prime} R^{\prime}\left[T, T^{-1}\right]$ shows that $L^{\prime}$ is an invertible ideal of $R^{\prime}$ and hence ( $R^{\prime}$ being semilocal) is a principal ideal, generated by, say, $r$. Thus we have $I R^{\prime}\left[T, T^{-1}\right]=\left(t, g_{1}, \ldots, g_{n}\right) R^{\prime}\left[T, T^{-1}\right]=r R^{\prime}\left[T, T^{-1}\right]$. Therefore $r R^{\prime}=\left(t, g_{1}(1), \ldots, g_{n}(1)\right) R^{\prime}=b R^{\prime}$. Hence without loss of generality we can assume that $r=b$.

Now we claim that there exists a finitely generated $R$-subalgebra $\widetilde{R}$ of $R^{\prime}$ such that

(1) $I \widetilde{R}\left[T, T^{-1}\right]=b \widetilde{R}\left[T, T^{-1}\right]$,

(2) $d^{l}(d=t / b) \in \mathscr{C}_{\widetilde{R} / R}$ for some positive integer $l$, where $\mathscr{C}_{\widetilde{R} / R}$ denotes the conductor ideal of $\widetilde{R}$ in $R$.

We will now complete the proof of the theorem by assuming this claim.

Since $\left(t=g_{0}, g_{1}, \ldots, g_{n}\right) \widetilde{R}\left[T, T^{-1}\right]=b \widetilde{R}\left[T, T^{-1}\right]$, we can write $b=$ $\sum_{i=0}^{n} h_{i} g_{i}$, where $h_{i} \in \widetilde{R}\left[T, T^{-1}\right]$. Then for $c \in \mathscr{C}=\mathscr{C}_{\widetilde{R} / R}$, we have $c b=$ $c \sum_{i=0}^{n} h_{i} g_{i}=\sum_{i=0}^{n}\left(c h_{i}\right) g_{i}$. Since $c \in \mathscr{C}$, we have $c h_{i} \in R\left[T, T^{-1}\right]$ and hence $c b \in I \cap R=t R$. This shows that $(b / t) \mathscr{C}=\mathscr{I}$ is an ideal of $R$. Clearly $\mathscr{I}$ is an ideal of $\widetilde{R}$ and hence $\mathscr{I} \subseteq \mathscr{C}$. This shows that $\mathscr{C} \subseteq d \mathscr{C}$ and therefore $\mathscr{C}=d \mathscr{C}$. Hence $\mathscr{C}=d \mathscr{C}=d^{2} \mathscr{C}=\cdots=d^{l} \mathscr{C} \subseteq d^{l} R \subseteq \mathscr{C}$. Therefore $\mathscr{C}=d^{l} R=d^{l+1} R$, which is absurd since $d$ is an element of the maximal ideal of $R$ which is a non-zero-divisor.

Therefore $I=t R\left[T, T^{-1}\right]$ as required.

Proof of the claim. Since $\left(t=g_{0}, g_{1}, \ldots, g_{n}\right) R^{\prime}\left[T, T^{-1}\right]=b R^{\prime}\left[T, T^{-1}\right], g_{i}=$ $b g_{i}^{\prime}(0 \leq i \leq n)$, where $g_{i}^{\prime} \in R^{\prime}\left[T, T^{-1}\right]$. In fact, since $g_{i} \in R[T]$ and $b$ is not a zero-divisor of $R^{\prime}$, we have $g_{i}^{\prime} \in R^{\prime}[T]$. Moreover $g_{0}^{\prime}=d$.

Let $K=b^{-1} I$. Then $K$ is an invertible $R\left[T, T^{-1}\right]$-submodule of $R^{\prime}\left[T, T^{-1}\right]$ generated by $\left\{g_{0}^{\prime}, g_{1}^{\prime}, \ldots, g_{n}^{\prime}\right\}$. Since $K R^{\prime}\left[T, T^{-1}\right]=R^{\prime}\left[T, T^{-1}\right]$, we have $K^{-1} \subseteq R^{\prime}\left[T, T^{-1}\right]$ and $K^{-1} R^{\prime}\left[T, T^{-1}\right]=R^{\prime}\left[T, T^{-1}\right]$.

Let $K^{-1}=\left(u_{0}, u_{1}, \ldots, u_{n}\right) R\left[T, T^{-1}\right]$, where $u_{i} \in R^{\prime}\left[T, T^{-1}\right]$ for $0 \leq i \leq$ $n$. Let $\widetilde{R}$ denote the finitely generated $R$-subalgebra of $R^{\prime}$ generated by the coefficients of $\left\{u_{i}\right\}_{i=0}^{n}$. Then $u_{i} \in \widetilde{R}\left[T, T^{-1}\right]$ for all $i$. Since $R^{\prime}$ is integral over $\widetilde{R}$ and $K^{-1} R^{\prime}\left[T, T^{-1}\right]=R^{\prime}\left[T, T^{-1}\right]$, we get that $K^{-1} \widetilde{R}\left[T, T^{-1}\right]=$ $\widetilde{R}\left[T, T^{-1}\right]$. This shows that $K \subseteq \widetilde{R}\left[T, T^{-1}\right]$ and $K \widetilde{R}\left[T, T^{-1}\right]=\widetilde{R}\left[T, T^{-1}\right]$. Since $K=b^{-1} I$, we get that $I \widetilde{R}\left[T, T^{-1}\right]=b \widetilde{R}\left[T, T^{-1}\right]$. This proves the first part of the claim.

Since $\widetilde{R}$ is generated as an $R$-algebra by coefficients of $u_{i}$, generators of $\widetilde{R}$ as an $R$-module can be chosen to be elements which are monomials in coefficients 
of $u_{i}$. Since $\widetilde{R}$ is a finite $R$-module, finitely many such monomials will generate $\widetilde{R}$ as an $R$-module. Since $d=g_{0}^{\prime} \in K$, we have $d u_{i} \in R\left[T, T^{-1}\right]$ for all $i$, $i=0,1, \ldots, n$. This shows that $d^{l} \widetilde{R} \subseteq R$ for some positive integer $l$. Thus the proof of the claim is complete.

Now assume that $R$ is a domain. If $I$ is extended from $R$ as a module, then by Lemma 2.4, $I=J R\left[T, T^{-1}\right]$, since $J=I \cap R \neq 0$. Therefore $J$ is an invertible ideal of $R$.

Remark 2.12. If $R$ is not necessarily a domain and if $I$ is extended from $R$ as a module, then under the hypothesis of the theorem we get, by Lemma 2.10, that there exists an element $f \in R\left[T, T^{-1}\right]$ such that $f I \cap R$ is an invertible ideal of $R$.

Theorem 2.13. Let $R$ be a reduced ring with only finitely many minimal prime ideals. Let $I$ be an invertible ideal of $R[T]$ such that $J=I \cap R$ contains a non-zero-divisor of $R$. Then $I$ is extended from $R$ as a module if and only if $J$ is an invertible ideal of $R$.

Proof. If $I$ is extended from $R$ then, by Lemma 2.8, $I=J R(T)$. Therefore it follows that $J$ is an invertible ideal of $R$. To prove the converse, without loss of generality, we can assume that $I$ is a proper ideal.

Let us assume that $J$ is an invertible ideal of $R$. Then as $I$ contains a non-zero-divisor, say $s$, of $R$, it is easy to see that the canonical epimorphism $I / T I \rightarrow I+(T) /(T) \simeq I / I \cap(T)$ is an isomorphism. This implies that $T$ is not a zero-divisor of $R[T] / I$. Therefore $I R\left[T, T^{-1}\right] \cap R[T]=I$. Now the ideal $I R\left[T, T^{-1}\right]$ is an invertible ideal of $R\left[T, T^{-1}\right]$ such that $I R\left[T, T^{-1}\right] \cap R=I \cap$ $R=J$. Therefore, by Theorem 2.11 , we get that $I R\left[T, T^{-1}\right]=J R\left[T, T^{-1}\right]$. This implies that for any $f \in I, T^{n} f \in J R[T]$ for some positive integer $n$. This shows that $f \in J R[T]$ and hence $I=J R[T]$ as required.

\section{ACKNOWLEDGMENT}

We thank Amit Roy and R. C. Cowsik for useful discussions.

\section{REFERENCES}

1. N. Bourbaki, Commutative algebra, Addison-Wesley, Reading, MA, 1972.

School of Mathematics, Tata Institute of Fundamental Research, Homi Bhabha ROAD, BOMBAY 400 005, INDIA

Current address, P. L. N. Varma: Mehta Research Insitute, 10 Kasturba Gandhi Marg, Allahabad211002, India

E-mail address, S. M. Bhatwadekar: SMB@TIFRVAX.BITNET 